\title{
Robust Finite-Time Tracking for Uncertain Linear Systems with Actuator Faults
}

\author{
Xinpeng Fang $(\mathbb{D}$, Huijin Fan $(\mathbb{D}$, and Lei Liu $(\mathbb{D}$ \\ School of Artificial Intelligence and Automation, National Key Laboratory of Science and Technology on Multispectral \\ Information Processing, Huazhong University of Science and Technology, Wuhan 430074, China \\ Correspondence should be addressed to Huijin Fan; ehjfan@hust.edu.cn
}

Received 22 July 2019; Revised 25 September 2019; Accepted 25 October 2019; Published 11 January 2020

Guest Editor: Baltazar Aguirre-Hernandez

Copyright $(92020$ Xinpeng Fang et al. This is an open access article distributed under the Creative Commons Attribution License, which permits unrestricted use, distribution, and reproduction in any medium, provided the original work is properly cited.

In this paper, a robust finite-time fault-tolerant control (FTC) scheme is developed for uncertain linear systems in the presence of actuator faults. Since the system uncertainties and actuator faults are unknown, the controller parameters are updated online by the adaptive laws without the need for fault detection and isolation. It is proved that the proposed state-feedback model reference adaptive finite-time FTC scheme can guarantee that the tracking error converges to a small neighborhood of the origin in finite time. An application example for an aircraft lateral-directional dynamic system is presented to show the effectiveness of the proposed control scheme.

\section{Introduction}

In a practical system, e.g., networked control system and flight control system, the actuator component usually suffers from a partial loss of effectiveness (LOE) or even a total loss of control (LOC) due to the increasing complexity of system itself and operating environment [1-5]. See for example, the rudder of an aircraft may experience a loss of gain in the control channel or be stuck in a fixed position. Such actuator faults may degrade the system performance, cause system instability, and even lead to catastrophic accidents. In order to improve system reliability and security, it is significantly important to consider the fault-tolerant control (FTC) problem. Numerous FTC strategies have been proposed which can be classified into two main types: (1) passive method and (2) active one. Passive FTC is actually a robust scheme by designing a fixed controller which ensures the closed-loop system be insensitive to specific preconsidered faults, see for example [6-9]. Unlike the passive control method, the active FTC method can guarantee the stability of the entire closed-loop system by adjusting the parameters or structure of the controller corresponding to the occurred fault. Therefore, compared with the passive control method, the active method is more flexible and practical. Fault detection and diagnosis (FDD) can provide fault information, for example, a sliding mode observer was developed in [10] for detecting and reconstructing actuator and sensor faults. Several active FTC methods based on FDD have been proposed in literatures, such as switching-based design [11], sliding mode control-based design [12, 13], pseudo-inverse approach [14], and model predictive control-based design [15]. It is noted that the performance of these active methods depends heavily on the accuracy of FDD.

On the contrary, adaptive FTC which is a kind of robust technique and a main class of active FTC has been widely used due to its flexibility and diversity in design and its ability to handle unknown actuator faults without the need for FDD modules [16-28]. By considering that the stuck fault is bounded, a robust adaptive FTC scheme was proposed for uncertain linear systems in [16]. In [17], a direct adaptive control scheme was designed to compensate for spacecraft systems with multiple actuator faults and inertia matrix uncertainties. A robust adaptive control strategy based on the generalized restricted potential function was proposed for linear systems in [18] to achieve the desired tracking error norm bound. By employing the adaptive backstepping technique, an adaptive control strategy was 
presented to deal with stochastic LOE faults for half-car active suspension systems in [19]. In [20, 21], two adaptive fault-tolerant controllers were developed with LOC faults only. By designing the auxiliary systems, the fault-tolerant constrained controllers were designed to achieve FTC in the presence of input amplitude saturation in [22, 23]. Moreover, as a promising adaptive control method, model reference adaptive control (MRAC) has been widely applied to guarantee the desired tracking performance and handle unknown actuator faults and system uncertainties. In $[29,30]$, state-feedback FTC schemes were presented to ensure the plant state asymptotically tracks the reference model state, and in [31,32], output tracking property was guaranteed by designing state-feedback MRAC schemes.

It is noted that the aforementioned control laws only guarantee the asymptotic stability of the system, that is, they only promise the stability of infinite time. However, in practice, we expect the desired system performance to be achieved in a finite time. For example, the actual attitude of the aircraft must recover the desired attitude within a finite time once fault occurring. Since finite-time control has a faster convergence rate, better robustness against uncertainties and disturbances, it has drawn a lot of attention. Therefore, designing a finite-time FTC scheme is of great significance. In [33-38], the issues of finite-time stability were investigated for fault-free cases. Two finite-time command filtered backstepping-based controllers were proposed in $[33,34]$, which guarantee the finite-time tracking property. In order to solve the problem of finitetime FTC, a number of control schemes have been developed in [39-41] and applied to spacecraft attitude control. In [39], a finite-time attitude controller was designed based on sliding mode control technology. A finite-time terminal sliding mode controller was proposed in [40] by using the reconstruction information of the adaptive terminal sliding mode observer. It is worth mentioning that the designed controllers in $[36,39,40]$ contain the sign functions, which may cause the undesired controller chattering problem. Therefore, it is more valuable to design the chattering-free finite-time FTC scheme. In addition, due to the inevitable existence of uncertainties, it is necessary and a key challenge to develop control strategies for uncertain systems. In [42], a robust adaptive hierarchical insensitive tracking controller was designed for linear systems with uncertainties in the system and input matrices.

Motivated by the above discussion, this paper will study the finite-time tracking control of uncertain linear systems with unknown actuator faults. The main contributions are given as follows:

(1) A robust model reference adaptive fault-tolerant tracking control scheme with finite-time convergence property for linear systems is developed to compensate for time-varying system matrix uncertainty and unknown actuator faults without requiring fault detection and isolation

(2) The unknown parameters caused by system uncertainty and actuator faults can be estimated by the designed tracking error-driven adaptive laws, which promises the adaptivity of the proposed controller

(3) The proposed control law is chattering-free, which is more practical for engineering applications

The rest of this paper is organized as follows. In Section 2, the problem to be addressed is formulated and some preliminaries are introduced. A robust adaptive finite-time FTC scheme is developed in Section 3. In Section 4, an application to an aircraft lateral-directional dynamic system is presented to illustrate the effectiveness of the proposed scheme. Finally, the conclusion is given in Section 5.

Notations. $\mathbb{R}^{n}$ represents the $n$-dimensional Euclidean space, while $\mathbb{R}^{n \times m}$ refers to the set of all $n \times m$ real matrices. $x \in \mathbb{R}^{n}$ denotes that $x$ is an $n$-dimensional vector, and $x \in \mathbb{R}^{n \times m}$ means that $x$ is an $n \times m$ matrix. $\|\cdot\|$ denotes the Euclidean norm of a vector or matrix. The superscript "T" stands for matrix transposition. $\operatorname{diag}\{. .$.$\} represents a block-$ diagonal matrix. $\lambda_{\min }(\cdot)$ and $\lambda_{\max }(\cdot)$ denote the minimum and maximum eigenvalues of a matrix, respectively. $I_{m}$ stands for the $m$-dimensional identity matrix.

\section{Preliminaries and Problem Statement}

\subsection{Preliminaries.}

Definition 1 (see [43]). Consider an autonomous dynamic system:

$$
\begin{aligned}
\dot{x} & =f(x), \\
f(0) & =0,
\end{aligned}
$$

where $f: U_{0} \longmapsto \mathbb{R}^{n}$ is continuous on an open neighborhood $U_{0}$ of the origin. The equilibrium $x=0$ of the system is uniformly finite-time stable if it is uniformly Lyapunov stable and for any initial condition $x_{0} \in U$ where $U \subset U_{0}$, if there is a settling time function $T: U \longmapsto(0, \infty)$, such that every solution $x\left(t, x_{0}\right)$ of system (1) satisfies $x\left(t, x_{0}\right) \in U \backslash\{0\}$ for $t \in\left[0, T\left(x_{0}\right)\right)$ with the properties: $\lim _{t \longrightarrow T\left(x_{0}\right)} x\left(t, x_{0}\right)=$ 0 and $x\left(t, x_{0}\right)=0, \forall t \geq T\left(x_{0}\right)$.

If $U=U_{0}=\mathbb{R}^{n}$, then the origin is a uniformly globally finite-time stable equilibrium.

Lemma 1 (see [44]). For $x_{j} \in \mathbb{R}, j=1,2, \ldots, n$, and $0<q<1$, the following relation holds:

$$
\left(\sum_{j=1}^{n}\left|x_{j}\right|\right)^{q} \leq \sum_{j=1}^{n}\left|x_{j}\right|^{q} .
$$

Lemma 2 (see [45]). For a positive definite matrix $A \in \mathbb{R}^{n \times n}$ and any $x \in \mathbb{R}^{n}$, the following inequality holds:

$$
\lambda_{\min }(A)\|x\|^{2} \leq x^{T} A x \leq \lambda_{\max }(A)\|x\|^{2} .
$$

Lemma 3 (see [36]). For any scalar $\delta_{0}>(1 / 2)$, matrices $K$, $\widehat{K}, \widetilde{K} \in \mathbb{R}^{n}$, and $\widetilde{K}=\widehat{K}-K$, the inequality in (4) holds: 


$$
-\widetilde{K}^{T} \widehat{K} \leq-\frac{2 \delta_{0}-1}{2 \delta_{0}} \widetilde{K}^{T} \widetilde{K}+\frac{\delta_{0}}{2} K^{T} K .
$$

Lemma 4 (see [46]). For some constants $a>0, b>0$, and $\mu>0$, the following relation holds:

$$
|y|^{a}|z|^{b} \leq \frac{a}{a+b} \mu|y|^{a+b}+\frac{b}{a+b} \mu^{-a / b}|z|^{a+b},
$$

where $y$ and $z$ are real variables.

Lemma 5 (see [36]). Consider the system $\dot{x}=f(x, u)$. If there exists a continuous positive definite function $V(x)$, real numbers $\eta>0,0<\alpha<1$, and $0<\bar{\psi}<\infty$, such that

$$
\dot{V}(x) \leq-\eta V^{\alpha}(x)+\bar{\psi},
$$

then the system $\dot{x}=f(x, u)$ is practical finite-time stable. The trajectories of the system can reach the set $\left\{x \mid V^{\alpha}(x) \leq(\bar{\psi} /(1-\imath) \eta)\right\}$ in a finite time $T_{r}$, which is defined as

$$
T_{r} \leq \frac{V^{1-\alpha}(0)}{\eta \iota(1-\alpha)}
$$

where $0<\iota<1$ and $V(0)$ is the initial value of $V(x)$.

2.2. System Model. Consider the linear systems with timevarying system matrix uncertainty described by

$$
\dot{x}(t)=(A+\Delta A(t)) x(t)+B u(t),
$$

where $x(t) \in \mathbb{R}^{n}$ is the state vector which can be available at every instant and $u(t)=\left[u_{1}(t), u_{2}(t), \ldots, u_{m}(t)\right]^{T} \in \mathbb{R}^{m}$ is the control input vector, which means that there are $m$ actuators in the system. $A \in \mathbb{R}^{n \times n}$ and $B \in \mathbb{R}^{n \times m}$ represent the nominal system and input matrices, respectively, where $B$ is full-row rank. $\Delta A(t)$ represents the time-varying system matrix uncertainty which satisfies the following condition:

$$
\Delta A(t)=B H(t)
$$

where $H(t)$ is an unknown continuous matrix function with $\|H(t)\| \leq \bar{h}$ and $\bar{h}$ is an unknown positive constant.

Remark 1. For a practical system, e.g., aircraft system [47] and industrial system [48], it can always be linearized and decoupled into the form of system (8). The full-row rank condition of $\mathrm{B}$ is a general requirement for the system, see for example $[18,49]$, which means that there must be enough control channels in the system, especially for actuator redundancy purpose. Then, the establishment of condition (9) is obvious, since any $n$-dimensional square matrix can be expressed in the form of (9) by a full-row rank matrix B.

2.3. Actuator Fault Model. The actuator fault model considered in this article is described as

$$
\begin{aligned}
u_{i}(t) & =\sigma_{i} v_{i}(t)+\rho_{i} \bar{u}_{i}(t), \\
\sigma_{i} \rho_{i} & =0, \quad i=1,2, \ldots, m,
\end{aligned}
$$

where $v_{i}(t)$ denotes the designed control input for the $i$ th actuator, $\bar{u}_{i}(t)$ represents the $i$ th actuator's uncontrollable time-varying fault, $\sigma_{i} \in[0,1]$ and $\rho_{i} \in\{0,1\}$ are unknown fault indicators for the $i$ th actuator, and $\sigma_{i}$ and $\bar{u}_{i}(t)$ are piecewise constant functions of time. More specifically

(1) The $i$ th actuator is fault-free when $\sigma_{i}=1$ and $\rho_{i}=0$

(2) The $i$ th actuator is partial LOE when $0<\sigma_{i}<1$ and $\rho_{i}=0$

(3) The $i$ th actuator corresponds to outage when $\sigma_{i}=0$ and $\rho_{i}=0$

(4) The ith actuator is stuck at $\bar{u}_{i}(t)$ when $\sigma_{i}=0$ and $\rho_{i}=1$

For convenience, the actual input vector $u(t)$ of system (8) can be formulated as

$$
u(t)=\sigma v(t)+\rho \bar{u}(t)
$$

where

$$
\begin{aligned}
\sigma & =\operatorname{diag}\left\{\sigma_{1}, \sigma_{2}, \ldots, \sigma_{m}\right\}, \\
\rho & =\operatorname{diag}\left\{\rho_{1}, \rho_{2}, \ldots, \rho_{m}\right\}, \\
v(t) & =\left[v_{1}(t), v_{2}(t), \ldots, v_{m}(t)\right]^{T}, \\
\bar{u}(t) & =\left[\bar{u}_{1}(t), \bar{u}_{2}(t), \ldots, \bar{u}_{m}(t)\right]^{T} .
\end{aligned}
$$

Remark 2. The compact fault model (11) includes normal (case 1), partial LOE faults (case 2) and total LOC faults (cases 3 and 4), which can represent most of the possible occurrences of an actuator in a practical system.

2.4. Control Objective. The reference model is given as

$$
\dot{x}_{m}(t)=A_{m} x_{m}(t)+B_{m} r_{m}(t),
$$

where $x_{m}(t) \in \mathbb{R}^{n}$ is the reference state vector which can be available at every instant, $r_{m}(t) \in \mathbb{R}^{m}$ is the bounded reference input, and $A_{m} \in \mathbb{R}^{n \times n}$ is a Hurwitz matrix, that is, there exist positive definite matrices $P, Q \in \mathbb{R}^{n \times n}$ such that

$$
A_{m}^{T} P+P A_{m}=-Q \text {. }
$$

Some assumptions are to be introduced.

Assumption 1. $\operatorname{rank}(B \sigma)=\operatorname{rank}(B)$.

Assumption 2. There exist $\bar{K}_{x} \in \mathbb{R}^{m \times n}$ and $\bar{K}_{r} \in \mathbb{R}^{m \times m}$ such that

$$
\begin{aligned}
A+B \bar{K}_{x} & =A_{m}, \\
B \bar{K}_{r} & =B_{m} .
\end{aligned}
$$

Remark 3. As discussed in [21], Assumption 1 is an actuator redundancy condition which ensures that the system 
remains capable to be stabilized even with actuator faults. In other words, the actuator faults satisfying Assumption 1 can be completely compensated by the proposed algorithm. Assumption 2 is a standard state-feedback state tracking matching condition, i.e., [29, 50]. In our scheme, Assumption 2 ensures that system (8) can match reference model (13) through state-feedback, so as to ensure the performance of state tracking. More specifically, according to Assumptions 1 and 2, it can be concluded that there exist $K_{x} \in \mathbb{R}^{m \times n}$ and $K_{r} \in \mathbb{R}^{m \times m}$ such that

$$
\begin{aligned}
A+B \sigma K_{x} & =A_{m}, \\
B \sigma K_{r} & =B_{m} .
\end{aligned}
$$

Lemma 6 (see [51]). The rank relation in Assumption 1 is a necessary and sufficient condition for the existence of a matrix $k_{1} \in \mathbb{R}^{m}$ such that

$$
B \sigma k_{1}=-B \rho \bar{u}
$$

Our control objective is to design a robust adaptive finitetime FTC scheme for system (8) in the presence of unknown actuator faults and system matrix uncertainty, such that the finite-time tracking is ensured for the given reference model (13).

\section{Robust Adaptive Finite-Time Fault-Tolerant Control Scheme}

In this section, a robust adaptive finite-time FTC scheme is developed to achieve the desired state tracking property in the presence of unknown actuator faults and system matrix uncertainty.

3.1. Controller Structure. Denote the tracking error $e=\left[e_{1}, e_{2}, \ldots, e_{n}\right]^{T}$ as

$$
e=x-x_{m}
$$

Let $\widehat{K}_{x}=\left[\widehat{K}_{x 1}, \widehat{K}_{x 2}, \ldots, \widehat{K}_{x m}\right]^{T} \in \mathbb{R}^{m \times n}, \widehat{K}_{r}=\left[\widehat{K}_{r 1}, \widehat{K}_{r 2}\right.$, $\left.\ldots, \widehat{K}_{r m}\right]^{T} \in \mathbb{R}^{m \times m}$, and $\widehat{k}_{1}=\left[\widehat{k}_{11}, \widehat{k}_{12}, \ldots, \widehat{k}_{1 m}\right]^{T} \in \mathbb{R}^{m}$ be the estimates of $K_{x}, K_{r}$, and $k_{1}$, respectively. Then, the finitetime FTC scheme in this article is constructed as

$$
v=\widehat{K}_{x} x+\widehat{K}_{r} r_{m}+\widehat{k}_{1}+k_{2}+k_{3}+k_{4} \text {, }
$$

where

$$
k_{2}=\frac{B^{T} P e e^{T} Q e}{2\left\|e^{T} P B\right\|^{2}+1},
$$

and $k_{3}$ and $k_{4} \in \mathbb{R}^{m}$ will be designed later.

By considering actuator faults (11), substituting (11) and (19) into (8), the closed-loop system can be formulated as $\dot{x}=(A+\Delta A) x+B \rho \bar{u}+B \sigma\left(\widehat{K}_{x} x+\widehat{K}_{r} r_{m}+\widehat{k}_{1}+k_{2}+k_{3}+k_{4}\right)$.

Together with (13), (16), (18), and (21) and using Lemma 6 , the tracking error system can be derived as

$$
\begin{aligned}
\dot{e}= & A_{m} e+B H x+B \sigma\left(\widehat{K}_{x}-K_{x}\right) x+B \sigma\left(\widehat{K}_{r}-K_{r}\right) r_{m} \\
& +B \sigma\left(\widehat{k}_{1}-k_{1}\right)+B \sigma\left(k_{2}+k_{3}+k_{4}\right) .
\end{aligned}
$$

Lemma 7 (see [16]). For the diagonal matrix $\sigma$ in (11), there exists a constant $0<\chi \leq 1$, such that

$$
\chi\left\|e^{T} P B\right\|^{2} \leq e^{T} P B \sigma B^{T} P e \leq\left\|e^{T} P B\right\|^{2} .
$$

Now, introducing two unknown positive scalars $\xi_{1}$ and $\xi_{2}$ as

$$
\begin{aligned}
& \xi_{1}=\frac{1-l_{1} \chi}{\chi}, \\
& \xi_{2}=\frac{\bar{h}}{\chi}
\end{aligned}
$$

where $0<l_{1}<1$ is a chosen constant and $\bar{h}$ is the upper bound of $H(t)$.

Then, term $k_{3}$ in (19) can be designed as

$$
k_{3}= \begin{cases}-\frac{l_{2} B^{T} P e\left(l_{1}+\widehat{\xi}_{1}\right)\left(e^{T} P e\right)^{\alpha}}{2\left\|e^{T} P B\right\|^{2}}, & \text { if }\left\|e^{T} P B\right\|>0, \\ 0_{m \times 1}, & \text { if }\left\|e^{T} P B\right\|=0,\end{cases}
$$

where $\widehat{\xi}_{1}$ is the estimate of unknown constant $\xi_{1}$ and $l_{2}>0$ and $0<\alpha<1$ are chosen constants.

Term $k_{4}$ in (19) is thus designed as

$$
k_{4}=-\frac{\widehat{\xi}_{2}^{2}\|x\|^{2} B^{T} P e}{\widehat{\xi}_{2}\|x\|\left\|B^{T} P e\right\|+l_{3}},
$$

where $\widehat{\xi}_{2}$ is the estimate of $\xi_{2}$ and $l_{3}$ is a chosen positive constant.

Remark 4. The designed controller (19) consists of six items: $\widehat{K}_{x} x, \widehat{K}_{r} r_{m}, \widehat{k}_{1}, k_{2}, k_{3}$, and $k_{4} . \widehat{K}_{x} x+\widehat{K}_{r} r_{m}$ is inherited from the typical state-feedback model reference controller structure. $\widehat{k}_{1}$ is the estimate of $k_{1}$ given in (17), which is to eliminate the effects of the stuck fault $\bar{u} . k_{2}$ is designed especially for stability analysis purpose. $k_{3}$ is actually an exponential term which is related to a finite-time control. $k_{4}$ is designed to eliminate the effects of uncertainty.

3.2. Adaptive Laws. To construct $v$, for $i=1,2, \ldots, m$, the adaptive laws to update $\widehat{K}_{x i}, \widehat{K}_{r i}, \widehat{k}_{1 i}, \widehat{\xi}_{1}$, and $\widehat{\xi}_{2}$ are chosen as

$$
\begin{aligned}
& \dot{\widehat{K}}_{x i}=-\Gamma_{i}\left(2 x e^{T} P b_{i}+\Lambda_{i} \widehat{K}_{x i}\right), \\
& \dot{\hat{K}}_{r i}=-\Upsilon_{i}\left(2 r_{m} e^{T} P b_{i}+\Theta_{i} \widehat{K}_{r i}\right), \\
& \dot{\widehat{k}}_{1 i}=-\mu_{i}\left(2 e^{T} P b_{i}+\tau_{i} \widehat{k}_{1 i}\right),
\end{aligned}
$$




$$
\begin{aligned}
& \dot{\vec{\xi}}_{1}=c_{1}\left(l_{2}\left(e^{T} P e\right)^{\alpha}-\varsigma_{1} \widehat{\xi}_{1}\right), \\
& \dot{\vec{\xi}}_{2}=c_{2}\left(2\left\|e^{T} P B\right\|\|x\|-\varsigma_{2} \widehat{\xi}_{2}\right),
\end{aligned}
$$

where $b_{i}$ represents the $i$ th column of $B, \Gamma_{i}, \Lambda_{i} \in \mathbb{R}^{n \times n}$, and $\Upsilon_{i}, \Theta_{i} \in \mathbb{R}^{m \times m}$ are chosen constant diagonal positive definite matrices, and $\mu_{i}, \tau_{i}>0$ and $c_{1}, c_{2}, \varsigma_{1}, \varsigma_{2}>0$ are chosen constants. The initial values of $\widehat{\xi}_{1}$ and $\widehat{\xi}_{2}$ are positive, that is, $\widehat{\xi}_{1}(0)>0$ and $\widehat{\xi}_{2}(0)>0$.

So far we have obtained the state-feedback model reference adaptive finite-time FTC scheme. For clarity, it is summarized in Appendix A.

\subsection{Stability Analysis}

Theorem 1. Consider the linear systems described by (8) with unknown actuator faults (11) and system matrix uncertainty, if Assumptions 1 and 2 are satisfied, the control signal in (19) updated by the adaptive laws (27)-(31) can guarantee that the tracking errors converge to a small neighborhood of the origin in finite time.

Proof. See Appendix B.

\section{Application: Aircraft Attitude Control}

In this section, simulation studies on a lateral-directional dynamic model of the F-18 high-angle-of-attack research vehicle (HARV) system [47] are given to illustrate the effectiveness of the proposed robust adaptive finite-time FTC scheme. To present the superiority of the proposed control scheme, the developed controller (19) is compared with the one given in [28].

4.1. F-18 HARV Model. The state variables for the linear lateral-directional dynamic model of the F-18 HARV system are side-slip angle $\beta(\mathrm{deg})$, roll rate $p(\mathrm{deg} / \mathrm{s})$, and yaw rate $r$ $(\mathrm{deg} / \mathrm{s})$, that is, $x=[\beta, p, r]^{T}$. The control input vector $u=\left[\delta_{D T}, \delta_{A I}, \delta_{R U}, \delta_{R T V}, \delta_{Y T V}\right]^{T}$, where $\delta_{D T}, \delta_{A I}, \delta_{R U}, \delta_{R T V}$, and $\delta_{Y T V}$ represent the differential tail deflection (deg), aileron deflection (deg), rudder deflection (deg), roll thrust vector deflection (deg), and yaw thrust vector deflection (deg), respectively.

As in [47], the linear plant is described by (8) with

$$
\begin{aligned}
A & =\left[\begin{array}{ccc}
-0.059 & 0.496 & -0.868 \\
-5.513 & -0.939 & 0.665 \\
0.068 & 0.026 & -0.104
\end{array}\right], \\
B & =\left[\begin{array}{ccccc}
0.006 & 0.006 & 0.004 & 0.000 & 0.090 \\
1.879 & 1.328 & 0.029 & 0.675 & 0.217 \\
-0.109 & -0.096 & -0.084 & 0.007 & -2.974
\end{array}\right] .
\end{aligned}
$$

The time-varying matrix $H(t)$ is given as

$$
H(t)=\left[\begin{array}{ccc}
0.1 \sin (0.1 t) & 0 & 0 \\
0 & 0.5 & 0 \\
0 & 0 & 1.6 \\
0 & 0 & 0.8 \\
e^{-2 t} & -1 & 0
\end{array}\right] .
$$

4.2. Simulation Scenarios. The reference model is chosen as (13) with

$$
\begin{aligned}
& A_{m}=\left[\begin{array}{ccc}
-0.153 & 0.486 & -0.778 \\
-6.310 & -2.971 & 0.331 \\
3.139 & 0.199 & -3.065
\end{array}\right], \\
& B_{m}=B .
\end{aligned}
$$

To demonstrate the effectiveness of the proposed adaptive finite-time FTC scheme, the following actuator faults are simulated:

$$
\begin{cases}u_{1}(t)=0.5 v_{1}(t), & \text { for } t \geq 10 \mathrm{~s}, \\ u_{2}(t)=0, & \text { for } 30 \mathrm{~s} \leq t<50 \mathrm{~s}, \\ u_{4}(t)=-5, & \text { for } 70 \mathrm{~s} \leq t<90 \mathrm{~s}, \\ u_{i}(t)=v_{i}(t), & i=1,2,3,4,5, \text { otherwise. }\end{cases}
$$

In (35), $u_{1}(t)=0.5 v_{1}(t)$ denotes the differential tail deflection $\delta_{D T}$ loses its $50 \%$ effectiveness; $u_{2}(t)=0$ denotes that the second actuator corresponds to outage, that is, the aileron deflection $\delta_{A I}$ stuck at the fixed value $0 \mathrm{deg}$; and $u_{4}(t)=-5$ denotes the roll thrust vector deflection $\delta_{R T V}$ cannot be influenced by control action $v_{4}(t)$ and stuck at the fixed value $-5 \mathrm{deg}$. Table 1 shows the fault indication matrices $\sigma$ and $\rho$ in different time intervals.

The initial conditions of the proposed controller (19) are given as follows:

$$
\begin{aligned}
x(0) & =[0.1,0.2,1.1]^{T}, \\
x_{m}(0) & =[-0.1,0.1,1]^{T}, \\
\widehat{K}_{x 1}(0) & =[-1,0,-1]^{T}, \\
\widehat{K}_{x 2}(0) & =[1,-1,1]^{T}, \\
\widehat{K}_{x 3}(0) & =[-1,-1,0]^{T}, \\
\widehat{K}_{x 4}(0) & =[0,-1,0]^{T}, \\
\widehat{K}_{x 5}(0) & =[-1,0,1]^{T}, \\
\widehat{\xi}_{1}(0) & =0.5, \\
\widehat{\xi}_{2}(0) & =1.8, \\
\widehat{K}_{r}(0) & =I_{5}, \\
\widehat{k}_{1 i}(0) & =0, \quad i=1,2,3,4,5 .
\end{aligned}
$$

The reference input signal $r_{m}=[0,0,0,0,2]^{T}$. In order to obtain a good FTC effect of the designed controller (19), after 
TABLE 1: Fault indication matrices $\sigma$ and $\rho$.

\begin{tabular}{lcc}
\hline Time interval & $\sigma$ & $\rho$ \\
\hline $0 \leq t<10 \mathrm{~s}$ & $\operatorname{diag}\{1,1,1,1,1\}$ & $\operatorname{diag}\{0,0,0,0,0\}$ \\
$10 \mathrm{~s} \leq t<30 \mathrm{~s}$ & $\operatorname{diag}\{0.5,1,1,1,1\}$ & $\operatorname{diag}\{0,0,0,0,0\}$ \\
$50 \mathrm{~s} \leq t<70 \mathrm{~s}$ & & $\operatorname{diag}\{0,0,0,0,0\}$ \\
and $t \geq 90 \mathrm{~s}$ & $\operatorname{diag}\{0.5,0,1,1,1\}$ & $\operatorname{diag}\{0,0,0,1,0\}$ \\
$30 \mathrm{~s} \leq t<50 \mathrm{~s}$ & $\operatorname{diag}\{0.5,1,1,0,1\}$ & \\
$70 \mathrm{~s} \leq t<90 \mathrm{~s}$ & &
\end{tabular}

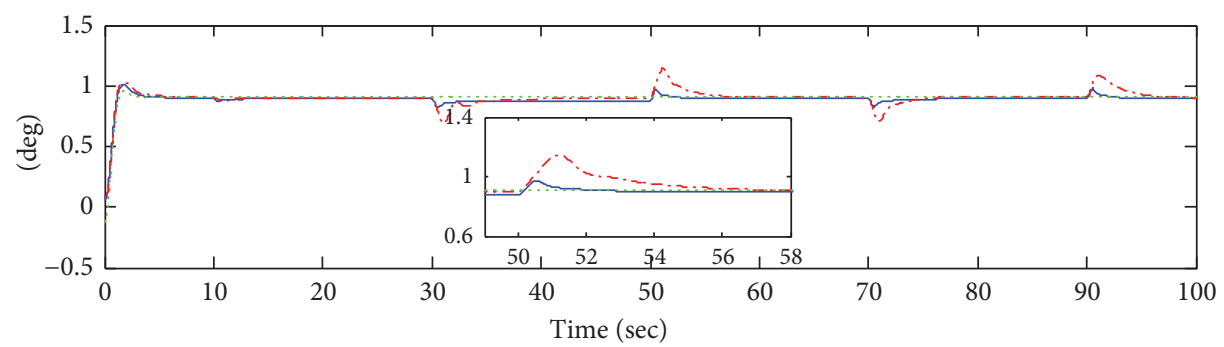

- $x_{1}(t)$ (controller (19))

...- $x_{1}(t)$ (controller in [28])

…. $x_{\mathrm{m} 1}(t)$

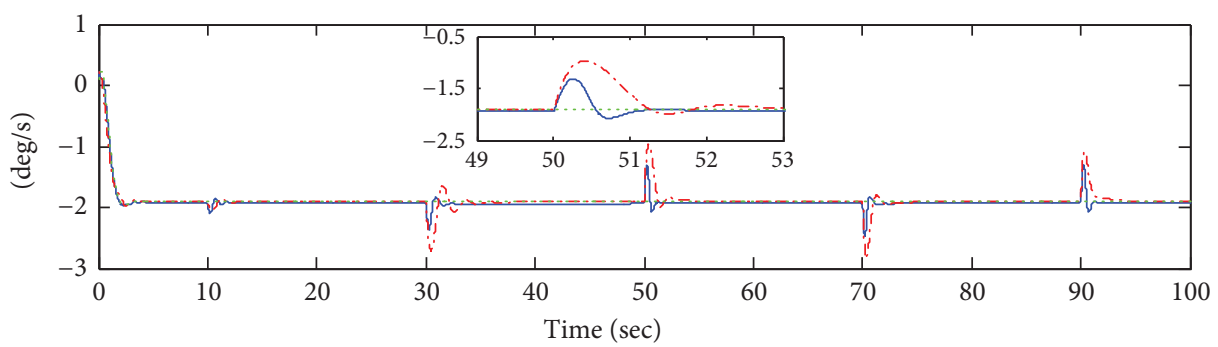

$-x_{2}(t)$ (controller (19))

-.. $x_{2}(t)$ (controller in [28])

$x_{\mathrm{m} 2}(t)$

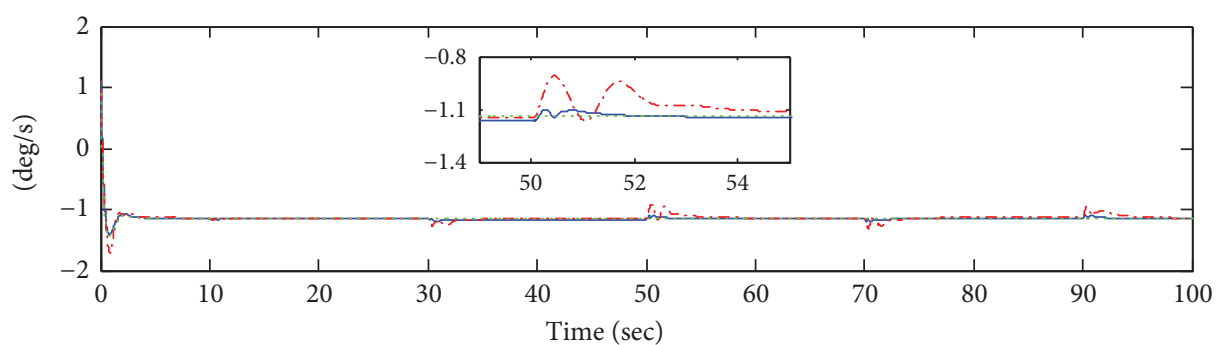

$x_{3}(t)($ controller $(19))$

... $x_{3}(t)$ (controller in [28])

$x_{\mathrm{m} 3}(t)$

Figure 1: Trajectories of plant state $x(t)$ and reference model state $x_{m}(t)$. 


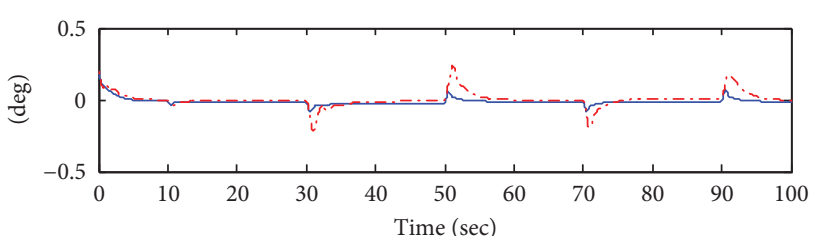

- $e_{1}(t)$ (controller (19))

-..- $e_{1}(t)$ (controller in [28])

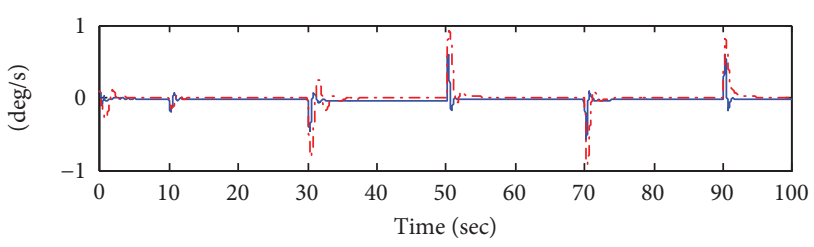

$e_{2}(t)$ (controller (19))

-..- $e_{2}(t)$ (controller in [28])

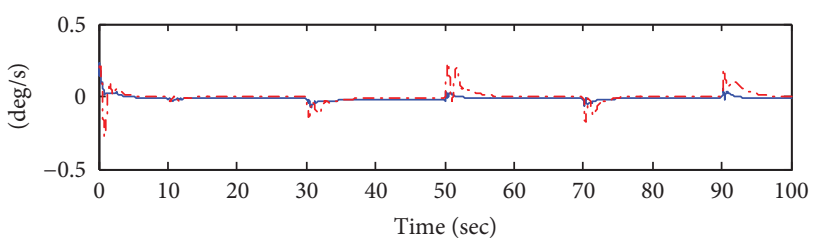

$-e_{3}(t)$ (controller (19))

$\cdot-\cdot-e_{3}(t)$ (controller in [28])

FIgURE 2: Trajectories of state tracking error $e(t)$.

repeatedly debugging, the controller parameters are chosen as

$$
\begin{aligned}
\Gamma_{i} & =I_{3}, \\
\Lambda_{i} & =0.1 I_{3}, \\
\Upsilon_{i} & =I_{5}, \\
\Theta_{i} & =0.1 I_{5}, \\
\mu_{i} & =1, \\
\tau_{i} & =0.1, \quad i=1,2,3,4,5, \\
c_{1} & =c_{2}=1, \\
\varsigma_{1} & =0.1, \\
\varsigma_{2} & =0.5, \\
l_{1} & =0.1, \\
l_{2} & =0.5, \\
l_{3} & =1, \\
\alpha & =0.8 .
\end{aligned}
$$

Although the parameters are chosen by trial and error, there are still some rules to be referred to. The larger the adaptive gains $\Gamma_{i}$ and $\Lambda_{i}$ in (27) are, the more drastic the change of $K_{x i}$ is, which leads to stronger control effect.
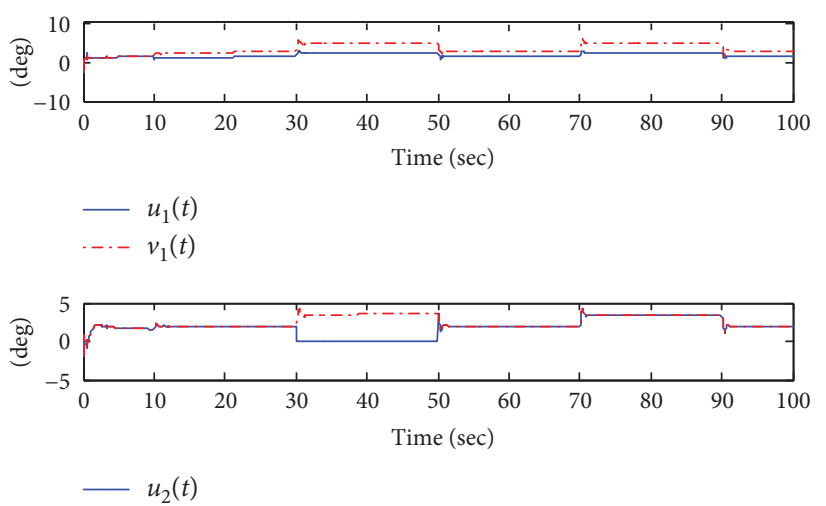

$\cdot-\cdot v_{2}(t)$

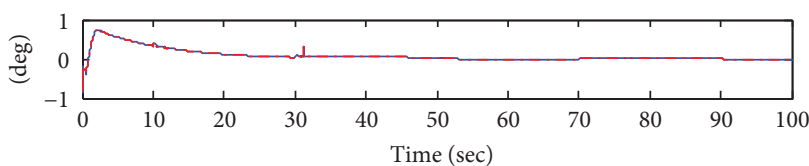

$-u_{3}(t)$

$\cdots v_{3}(t)$

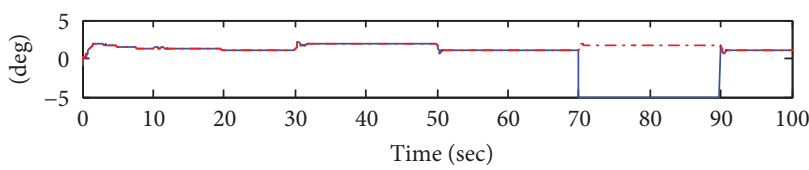

$-u_{4}(t)$

$\cdots v_{4}(t)$

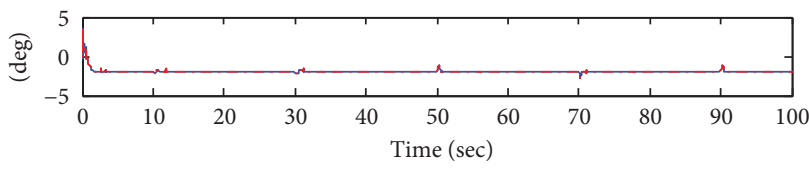

$-u_{5}(t)$

$\cdot \cdot \cdot-v_{5}(t)$

Figure 3: Actual control signal $u(t)$ and designed control signal $v(t)$ of controller (19).

Similar phenomenon lies in (28)-(31). However, too-large adaptive gains will affect the smoothness of the controller. $l_{1}$ and $l_{2}$ have direct impacts on $k_{3}$, which means that they will affect the strength of the controller directly. $l_{3}$ is a constant relating to the smoothness of the controller, while a large $l_{3}$ will lead to a large $\bar{\psi}$ and thus reduce the finite-time convergence accuracy. If $\alpha$ chosen by the designer is too small, then $k_{3}$ will exhibit a fast rate of change when the tracking error turns small, which is not expected in a practical system. In sum, to obtain a more practical FTC performance, the designers should choose the parameters with a compromise among the convergence rate, convergence accuracy, and the amplitude and smoothness of the control signals.

Through trial and error, the initial conditions and parameters of the controller given in [28] are chosen as follows to obtain a good FTC effect: 

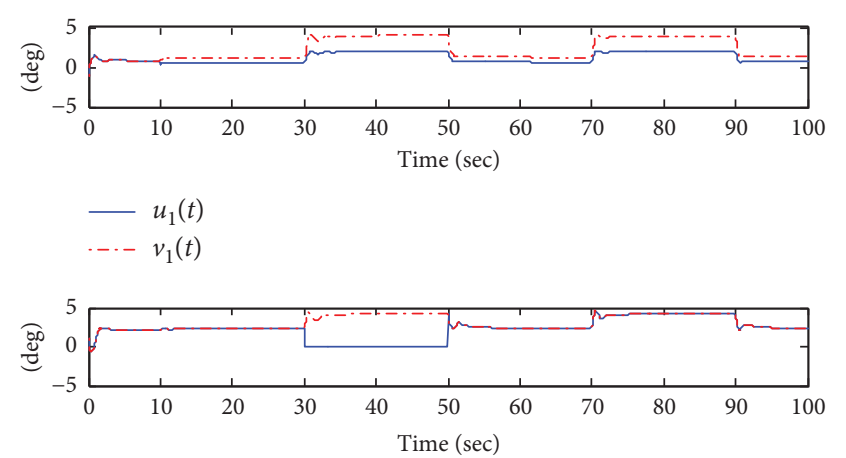

$-u_{2}(t)$

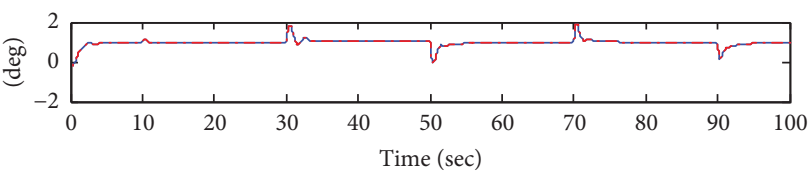

$-u_{3}(t)$

$\cdot-\cdot-v_{3}(t)$
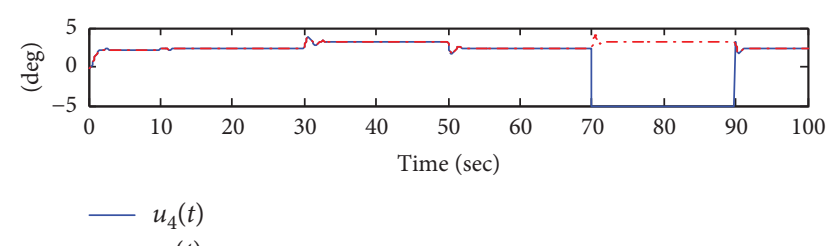

$\cdot-\cdot v_{4}(t)$

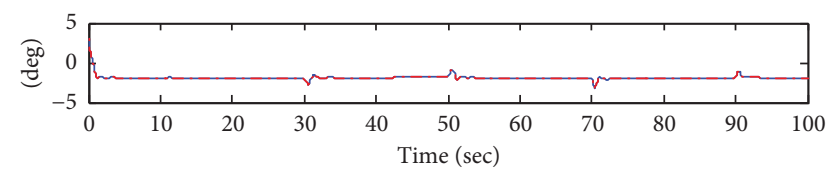

$-u_{5}(t)$

Figure 4: Actual control signal $u(t)$ and designed control signal $v(t)$ of the controller given in [28].

$$
\begin{aligned}
\widehat{H}_{11}(0) & =[-1,0,-1]^{T}, \\
\widehat{H}_{12}(0) & =[1,-1,1]^{T}, \\
\widehat{H}_{13}(0) & =[-1,-1,0]^{T}, \\
\widehat{H}_{14}(0) & =[0,-1,0]^{T}, \\
\widehat{H}_{15}(0) & =[-1,0,1]^{T}, \\
\widehat{k}_{1}(0) & =\widehat{k}_{2}(0)=\widehat{k}_{3}(0)=0, \\
\widehat{M}_{1}(0) & =I_{5}, \\
\eta & =1, \\
\gamma_{1} & =\gamma_{2}=\gamma_{3}=0.6, \\
L & =0, \\
\Gamma_{i} & =0.6, \\
\Lambda_{i} & =0.6, \quad i=1,2,3,4,5 .
\end{aligned}
$$

4.3. Simulation Results. Figure 1 shows the plant state $x(t)$ and reference model state $x_{m}(t)$ by using the proposed controller (19) and the one given in [28]; Figure 2 shows the state tracking error $e(t)$ by using controller (19) and the one given in [28]; Figure 3 shows the actual control signal $u(t)$ and designed control signal $v(t)$ of controller (19); and Figure 4 shows the actual control signal $u(t)$ and designed control signal $v(t)$ of the controller given in [28].

By using the proposed controller (19), it can be seen that (1) during $0 \leq t<10 \mathrm{~s}$, there is only system matrix uncertainty and no fault, and the tracking performance can be guaranteed in finite time; (2) whenever a new fault occurs at 10,30 , and $70 \mathrm{~s}$ and the fault recovers at 50 and $90 \mathrm{~s}$, after the transient response, the tracking performance recovers. Similar conclusions can be obtained when using the controller given in [28], while it only guarantees the asymptotic tracking property, that is, it only promises the stability of infinite time. It is obvious that the proposed robust finitetime FTC scheme possesses a better transient response and faster convergence rate.

Summarizing all the above results, it is clear that the proposed robust adaptive finite-time FTC scheme can effectively ensure the state practical tracking property in finite time in the presence of unknown actuator faults and system matrix uncertainty.

\section{Conclusions}

In this article, a state-feedback model reference adaptive finite-time FTC scheme has been developed for linear systems in the presence of unknown actuator faults and system matrix uncertainty. The designed robust controller updated by the adaptive laws ensures that the tracking errors converge to a small neighborhood of the origin in finite time. Finally, the performance of the proposed control scheme is illustrated by simulation results for a lateral-directional dynamic model of the F-18 HARV system.

Our future work will focus on the control problem for the system under practical constrains, such as actuator amplitude saturation, rate limitation, and input matrix uncertainty.

\section{Appendix}

\section{A. The Control Algorithm}

The proposed control algorithm is summarized as follows:

Step 1 . Give the initial state $x(0), x_{m}(0)$, the reference model input $r_{m}(t)$, and initial values of parameter estimates $\widehat{K}_{x}(0), \widehat{K}_{r}(0), \widehat{k}_{1}(0), \widehat{\xi}_{1}(0)$, and $\widehat{\xi}_{2}(0)$.

Step 2. For $i=1,2, \ldots, m$, choose the following adaptive gains:

$$
\begin{array}{ccccc}
\Gamma_{i}, & \Lambda_{i}, & \Upsilon_{i}, & \Theta_{i}, & \mu_{i}, \\
\tau_{i}, & c_{1}, & c_{2}, & \varsigma_{1}, & \varsigma_{2},
\end{array}
$$


and constants

$$
l_{1}, \quad l_{2}, \quad l_{3}, \quad \alpha
$$

Step 3. Estimate the unknown parameters by adaptive laws:

$$
\begin{aligned}
& \dot{\hat{K}}_{x i}=-\Gamma_{i}\left(2 x e^{T} P b_{i}+\Lambda_{i} \widehat{K}_{x i}\right), \\
& \dot{\hat{K}}_{r i}=-\Upsilon_{i}\left(2 r_{m} e^{T} P b_{i}+\Theta_{i} \widehat{K}_{r i}\right), \\
& \dot{\hat{k}}_{1 i}=-\mu_{i}\left(2 e^{T} P b_{i}+\tau_{i} \widehat{k}_{1 i}\right), \\
& \dot{\vec{\xi}}_{1}=c_{1}\left(l_{2}\left(e^{T} P e\right)^{\alpha}-\varsigma_{1} \widehat{\xi}_{1}\right), \\
& \dot{\vec{\xi}}_{2}=c_{2}\left(2\left\|e^{T} P B\right\|\|x\|-\varsigma_{2} \widehat{\xi}_{2}\right) .
\end{aligned}
$$

Step 4. Obtain the controller:

$$
v=\widehat{K}_{x} x+\widehat{K}_{r} r_{m}+\widehat{k}_{1}+k_{2}+k_{3}+k_{4},
$$

where

$$
\begin{aligned}
& k_{2}=\frac{B^{T} P e e^{T} Q e}{2\left\|e^{T} P B\right\|^{2}+1}, \\
& k_{3}= \begin{cases}\frac{l_{2} B^{T} P e\left(l_{1}+\widehat{\xi}_{1}\right)\left(e^{T} P e\right)^{\alpha}}{2\left\|e^{T} P B\right\|^{2}}, & \text { if }\left\|e^{T} P B\right\|>0, \\
0_{m \times 1}, & \text { if }\left\|e^{T} P B\right\|=0,\end{cases} \\
& k_{4}=-\frac{\widehat{\xi}_{2}^{2}\|x\|^{2} B^{T} P e}{\widehat{\xi}_{2}\|x\|\left\|B^{T} P e\right\|+l_{3} .}
\end{aligned}
$$

\section{B. Proof of Theorem 1}

Proof. Construct the Lyapunov function as

$$
\begin{aligned}
V= & e^{T} P e+\frac{1}{2} \sum_{i=1}^{m} \sigma_{i} \widetilde{K}_{x i}^{T} \Gamma_{i}^{-1} \widetilde{K}_{x i}+\frac{1}{2} \sum_{i=1}^{m} \sigma_{i} \widetilde{K}_{r i}^{T} \Upsilon_{i}^{-1} \widetilde{K}_{r i} \\
& +\frac{1}{2} \sum_{i=1}^{m} \sigma_{i} \mu_{i}^{-1} \widetilde{k}_{1 i}^{2}+\frac{1}{2} c_{1}^{-1} \chi \widetilde{\xi}_{1}^{2}+\frac{1}{2} c_{2}^{-1} \chi \widetilde{\xi}_{2}^{2},
\end{aligned}
$$

where $\quad \widetilde{K}_{x i}=\widehat{K}_{x i}-K_{x i}, \quad \widetilde{K}_{r i}=\widehat{K}_{r i}-K_{r i}, \quad \widetilde{k}_{1 i}=\widehat{k}_{1 i}-k_{1 i}$, $\widetilde{\xi}_{1}=\widehat{\xi}_{1}-\xi_{1}$, and $\widetilde{\xi}_{2}=\widehat{\xi}_{2}-\xi_{2}$.

The time derivative of $V$ can be derived as follows:

$$
\begin{aligned}
\dot{V}= & 2 e^{T} P\left[A_{m} e+B H x+B \sigma \widetilde{K}_{x} x+B \sigma \widetilde{K}_{r} r_{m}+B \sigma \widetilde{k}_{1}\right. \\
& \left.+B \sigma\left(k_{2}+k_{3}+k_{4}\right)\right]+\sum_{i=1}^{m} \sigma_{i} \widetilde{K}_{x i}^{T} \Gamma_{i}^{-1} \dot{\hat{K}}_{x i}+\sum_{i=1}^{m} \sigma_{i} \widetilde{K}_{r i}^{T} \Upsilon_{i}^{-1} \dot{\hat{K}}_{r i} \\
& +\sum_{i=1}^{m} \sigma_{i} \mu_{i}^{-1} \widetilde{k}_{1 i} \dot{\hat{k}}_{1 i}+c_{1}^{-1} \chi \widetilde{\xi}_{1} \dot{\vec{\xi}}_{1}+c_{2}^{-1} \chi \widetilde{\xi}_{2} \dot{\hat{\xi}}_{2} .
\end{aligned}
$$

Now, discuss the term $2 e^{T} P B \sigma k_{3}$ in two cases.

Case 1. If $\left\|e^{T} P B\right\|>0$, by using Lemma 7 , it has

$$
\begin{aligned}
2 e^{T} P B \sigma k_{3} & =-\frac{2 l_{2} e^{T} P B \sigma B^{T} P e\left(l_{1}+\widehat{\xi}_{1}\right)\left(e^{T} P e\right)^{\alpha}}{2\left\|e^{T} P B\right\|^{2}} \\
& \leq-\chi l_{2}\left(l_{1}+\widehat{\xi}_{1}\right)\left(e^{T} P e\right)^{\alpha} .
\end{aligned}
$$

Case 2. If $\left\|e^{T} P B\right\|=0$, due to the fact of $B$ being full-row rank, for positive definite matrix $P$, it follows that $P B$ is fullrow rank, that is, in this case $e=0_{n \times 1}$, hence,

$$
2 e^{T} P B \sigma k_{3}=-\chi l_{2}\left(l_{1}+\widehat{\xi}_{1}\right)\left(e^{T} P e\right)^{\alpha}=0 .
$$

Combining the above discussions, we get

$$
\begin{aligned}
2 e^{T} P B \sigma k_{3} \leq & -\chi l_{2}\left(l_{1}+\widehat{\xi}_{1}\right)\left(e^{T} P e\right)^{\alpha} \\
= & -l_{2}\left(e^{T} P e\right)^{\alpha}+l_{2}\left(1-l_{1} \chi\right)\left(e^{T} P e\right)^{\alpha} \\
& -l_{2} \chi \widehat{\xi}_{1}\left(e^{T} P e\right)^{\alpha} .
\end{aligned}
$$

Substituting (20), (26), and (B.5) into (B.2), we obtain

$$
\begin{aligned}
\dot{V} \leq & -l_{2}\left(e^{T} P e\right)^{\alpha}+2 e^{T} P A_{m} e+2\left\|e^{T} P B\right\|\|H\|\|x\| \\
& +2 e^{T} P B \sigma \widetilde{K}_{x} x+2 e^{T} P B \sigma \widetilde{K}_{r} r_{m}+2 e^{T} P B \sigma \widetilde{k}_{1} \\
& +l_{2}\left(1-l_{1} \chi\right)\left(e^{T} P e\right)^{\alpha}-l_{2} \chi \widehat{\xi}_{1}\left(e^{T} P e\right)^{\alpha} \\
& -\frac{2 \widehat{\xi}_{2}^{2}\|x\|^{2} e^{T} P B \sigma B^{T} P e}{\widehat{\xi}_{2}\|x\|\left\|B^{T} P e\right\|+l_{3}}+\frac{2 e^{T} P B \sigma B^{T} P e e^{T} Q e}{2\left\|e^{T} P B\right\|^{2}+1} \\
& +\sum_{i=1}^{m} \sigma_{i} \widetilde{K}_{x i}^{T} \Gamma_{i}^{-1} \dot{\hat{K}}_{x i}+\sum_{i=1}^{m} \sigma_{i} \widetilde{K}_{r i}^{T} \Upsilon_{i}^{-1} \dot{\hat{K}}_{r i} \\
& +\sum_{i=1}^{m} \sigma_{i} \mu_{i}^{-1} \widetilde{k}_{1 i} \dot{\hat{\kappa}}_{1 i}+c_{1}^{-1} \chi \widetilde{\xi}_{1} \dot{\vec{\xi}}_{1}+c_{2}^{-1} \chi \widetilde{\xi}_{2} \dot{\vec{\xi}}_{2} .
\end{aligned}
$$


According to the definition of $\xi_{1}$ and $\xi_{2}$ in (24), it has

$$
\begin{aligned}
l_{2}\left(1-l_{1} \chi\right)\left(e^{T} P e\right)^{\alpha} & =l_{2} \chi \xi_{1}\left(e^{T} P e\right)^{\alpha}, \\
2\left\|e^{T} P B\right\|\|H\|\|x\| & \leq 2 \bar{h}\left\|e^{T} P B\right\|\|x\| \\
& =2 \chi \xi_{2}\left\|e^{T} P B\right\|\|x\| .
\end{aligned}
$$

Then, using Lemma 7 and substituting (B.7) and (B.8) into (B.6) yields that

$$
\begin{aligned}
\dot{V} \leq & -l_{2}\left(e^{T} P e\right)^{\alpha}+e^{T}\left(A_{m}^{T} P+P A_{m}\right) e+2 \chi \xi_{2}\left\|e^{T} P B\right\|\|x\| \\
& +2 e^{T} P B \sigma \widetilde{K}_{x} x+2 e^{T} P B \sigma \widetilde{K}_{r} r_{m}+2 e^{T} P B \sigma \widetilde{k}_{1} \\
& -l_{2} \chi \widetilde{\xi}_{1}\left(e^{T} P e\right)^{\alpha}-\frac{2 \chi \widehat{\xi}_{2}^{2}\|x\|^{2}\left\|e^{T} P B\right\|^{2}}{\widehat{\xi}_{2}\|x\|\left\|B^{T} P e\right\|+l_{3}} \\
& +\frac{2\left\|e^{T} P B\right\|^{2} e^{T} Q e}{2\left\|e^{T} P B\right\|^{2}+1}+\sum_{i=1}^{m} \sigma_{i} \widetilde{K}_{x i}^{T} \Gamma_{i}^{-1} \dot{\hat{K}}_{x i} \\
& +\sum_{i=1}^{m} \sigma_{i} \widetilde{K}_{r i}^{T} \Upsilon_{i}^{-1} \dot{\hat{K}}_{r i}+\sum_{i=1}^{m} \sigma_{i} \mu_{i}^{-1} \widetilde{k}_{1 i} \dot{\hat{k}}_{1 i} \\
& +c_{1}^{-1} \chi \widetilde{\xi}_{1} \dot{\vec{\xi}}_{1}+c_{2}^{-1} \chi \widetilde{\xi}_{2} \dot{\vec{\xi}}_{2} .
\end{aligned}
$$

Substituting (14) and adaptive laws in (27)-(31) into (B.9) yields

$$
\begin{aligned}
\dot{V} \leq & -l_{2}\left(e^{T} P e\right)^{\alpha}-e^{T} Q e+2 \chi \xi_{2}\left\|e^{T} P B\right\|\|x\| \\
& +2 e^{T} P B \sigma \widetilde{K}_{x} x+2 e^{T} P B \sigma \widetilde{K}_{r} r_{m}+2 e^{T} P B \sigma \widetilde{k}_{1} \\
& -l_{2} \chi \widetilde{\xi}_{1}\left(e^{T} P e\right)^{\alpha}-\frac{2 \chi \widehat{\xi}_{2}^{2}\|x\|^{2}\left\|e^{T} P B\right\|^{2}}{\widehat{\xi}_{2}\|x\|\left\|B^{T} P e\right\|+l_{3}} \\
& +\frac{2\left\|e^{T} P B\right\|^{2} e^{T} Q e}{2\left\|e^{T} P B\right\|^{2}+1}-2 \sum_{i=1}^{m} \sigma_{i} \widetilde{K}_{x i}^{T} x e^{T} P b_{i} \\
& -\sum_{i=1}^{m} \sigma_{i} \widetilde{K}_{x i}^{T} \Lambda_{i} \widehat{K}_{x i}-2 \sum_{i=1}^{m} \sigma_{i} \widetilde{K}_{r i}^{T} r_{m} e^{T} P b_{i} \\
& -\sum_{i=1}^{m} \sigma_{i} \widetilde{K}_{r i}^{T} \Theta_{i} \widehat{K}_{r i}-2 \sum_{i=1}^{m} \sigma_{i} \widetilde{k}_{1 i} e^{T} P b_{i} \\
& -\sum_{i=1}^{m} \sigma_{i} \widetilde{k}_{1 i} \tau_{i} \widehat{k}_{1 i}+l_{2} \chi \widetilde{\xi}_{1}\left(e e^{T} P e\right)^{\alpha}-\varsigma_{1} \chi \widetilde{\xi}_{1} \widehat{\xi}_{1} \\
& +2 \chi \widetilde{\xi}_{2}\left\|e^{T} P B\right\|\|x\|-\varsigma_{2} \chi \widetilde{\xi}_{2} \widehat{\xi}_{2}
\end{aligned}
$$

$$
\begin{aligned}
= & -l_{2}\left(e^{T} P e\right)^{\alpha}-e^{T} Q e-\sum_{i=1}^{m} \sigma_{i} \widetilde{K}_{x i}^{T} \Lambda_{i} \widehat{K}_{x i}-\varsigma_{1} \chi \widetilde{\xi}_{1} \widehat{\xi}_{1} \\
& -\sum_{i=1}^{m} \sigma_{i} \widetilde{K}_{r i}^{T} \Theta_{i} \widehat{K}_{r i}-\sum_{i=1}^{m} \sigma_{i} \widetilde{k}_{1 i} \tau_{i} \widehat{k}_{1 i}-\varsigma_{2} \chi \widetilde{\xi}_{2} \widehat{\xi}_{2} \\
& +\frac{2 \chi l_{3} \widehat{\xi}_{2}\|x\|\left\|e^{T} P B\right\|}{\widehat{\xi}_{2}\|x\|\left\|B^{T} P e\right\|+l_{3}}+\frac{2\left\|e^{T} P B\right\|^{2} e^{T} Q e}{2\left\|e^{T} P B\right\|^{2}+1} .
\end{aligned}
$$

Then, according to the inequality

$$
0 \leq \frac{c d}{c+d} \leq c, \quad \forall c, d \geq 0, c+d \neq 0
$$

it has

$$
\begin{gathered}
\frac{2 \chi l_{3} \widehat{\xi}_{2}\|x\|\left\|e^{T} P B\right\|}{\widehat{\xi}_{2}\|x\|\left\|B^{T} P e\right\|+l_{3}} \leq 2 \chi l_{3}, \\
\frac{2\left\|e^{T} P B\right\|^{2} e^{T} Q e}{2\left\|e^{T} P B\right\|^{2}+1} \leq e^{T} Q e .
\end{gathered}
$$

Then, substituting (B.12) and (B.13) into (B.10), we obtain

$$
\begin{aligned}
\dot{V} \leq & -l_{2}\left(e^{T} P e\right)^{\alpha}-\sum_{i=1}^{m} \sigma_{i} \widetilde{K}_{x i}^{T} \Lambda_{i} \widehat{K}_{x i}-\sum_{i=1}^{m} \sigma_{i} \widetilde{K}_{r i}^{T} \Theta_{i} \widehat{K}_{r i} \\
& -\sum_{i=1}^{m} \sigma_{i} \widetilde{k}_{1 i} \tau_{i} \widehat{k}_{1 i}-\varsigma_{1} \chi \widetilde{\xi}_{1} \widehat{\xi}_{1}-\varsigma_{2} \chi \widetilde{\xi}_{2} \widehat{\xi}_{2}+2 \chi l_{3} .
\end{aligned}
$$

From Lemma 3, for any $\vartheta_{i}, \gamma_{i}, \omega_{i}>(1 / 2), i=1,2, \ldots, m$, and $\varrho_{1}, \varrho_{2}>(1 / 2)$, one has

$$
\begin{aligned}
-\widetilde{K}_{x i}^{T} \widehat{K}_{x i} \leq-\frac{2 \vartheta_{i}-1}{2 \vartheta_{i}} \widetilde{K}_{x i}^{T} \widetilde{K}_{x i}+\frac{\vartheta_{i}}{2} K_{x i}^{T} K_{x i}, \\
-\widetilde{K}_{r i}^{T} \widehat{K}_{r i} \leq-\frac{2 \gamma_{i}-1}{2 \gamma_{i}} \widetilde{K}_{r i}^{T} \widetilde{K}_{r i}+\frac{\gamma_{i}}{2} K_{r i}^{T} K_{r i}, \\
-\widetilde{k}_{1 i} \widehat{k}_{1 i} \leq-\frac{2 \omega_{i}-1}{2 \omega_{i}} \widetilde{k}_{1 i}^{2}+\frac{\omega_{i}}{2} k_{1 i}^{2}, \\
-\widetilde{\xi}_{1} \widehat{\xi}_{1} \leq-\frac{2 \varrho_{1}-1}{2 \varrho_{1}} \widetilde{\xi}_{1}^{2}+\frac{\varrho_{1}}{2} \xi_{1}^{2}, \\
-\widetilde{\xi}_{2} \widehat{\xi}_{2} \leq-\frac{2 \varrho_{2}-1}{2 \varrho_{2}} \widetilde{\xi}_{2}^{2}+\frac{\varrho_{2}}{2} \xi_{2}^{2} .
\end{aligned}
$$

Now, substituting (B.15)-(B.19) into (B.14), we have 


$$
\begin{aligned}
\dot{V} \leq & -l_{2}\left(e^{T} P e\right)^{\alpha}-\sum_{i=1}^{m}\left(\frac{2 \vartheta_{i}-1}{2 \vartheta_{i}} \sigma_{i} \widetilde{K}_{x i}^{T} \Lambda_{i} \widetilde{K}_{x i}\right)^{\alpha} \\
& -\sum_{i=1}^{m}\left(\frac{2 \gamma_{i}-1}{2 \gamma_{i}} \sigma_{i} \widetilde{K}_{r i}^{T} \Theta_{i} \widetilde{K}_{r i}\right)^{\alpha}-\sum_{i=1}^{m}\left(\frac{2 \omega_{i}-1}{2 \omega_{i}} \sigma_{i} \tau_{i} \widetilde{k}_{1 i}^{2}\right)^{\alpha} \\
& -\left(\frac{2 \varrho_{1}-1}{2 \varrho_{1}} \varsigma_{1} \chi \widetilde{\xi}_{1}^{2}\right)^{\alpha}-\left(\frac{2 \varrho_{2}-1}{2 \varrho_{2}} \varsigma_{2} \chi \widetilde{\xi}_{2}^{2}\right)^{\alpha} \\
& +\sum_{i=1}^{m}\left(\frac{2 \vartheta_{i}-1}{2 \vartheta_{i}} \sigma_{i} \widetilde{K}_{x i}^{T} \Lambda_{i} \widetilde{K}_{x i}\right)^{\alpha}-\sum_{i=1}^{m} \frac{2 \vartheta_{i}-1}{2 \vartheta_{i}} \sigma_{i} \widetilde{K}_{x i}^{T} \Lambda_{i} \widetilde{K}_{x i} \\
& +\sum_{i=1}^{m}\left(\frac{2 \gamma_{i}-1}{2 \gamma_{i}} \sigma_{i} \widetilde{K}_{r i} \Theta_{i} \widetilde{K}_{r i}\right)^{\alpha}-\sum_{i=1}^{m} \frac{2 \gamma_{i}-1}{2 \gamma_{i}} \sigma_{i} \widetilde{K}_{r i}^{T} \Theta_{i} \widetilde{K}_{r i} \\
& +\sum_{i=1}^{m}\left(\frac{2 \omega_{i}-1}{2 \omega_{i}} \sigma_{i} \tau_{i} \widetilde{k}_{1 i}^{2}\right)^{\alpha}-\sum_{i=1}^{m} \frac{2 \omega_{i}-1}{2 \omega_{i}} \sigma_{i} \tau_{i} \widetilde{k}_{1 i}^{2} \\
& +\left(\frac{2 \varrho_{1}-1}{2 \varrho_{1}} \varsigma_{1} \chi \widetilde{\xi}_{1}^{2}\right)^{\alpha}-\frac{2 \varrho_{1}-1}{2 \varrho_{1}} \varsigma_{1} \chi \widetilde{\xi}_{1}^{2} \\
& +\left(\frac{2 \varrho_{2}-1}{2 \varrho_{2}} \varsigma_{2} \chi \widetilde{\xi}_{2}^{2}\right)^{\alpha}-\frac{2 \varrho_{2}-1}{2 \varrho_{2}} \varsigma_{2} \chi \widetilde{\xi}_{2}^{2} \\
& +\sum_{i=1}^{m} \frac{\vartheta_{i}}{2} K_{x i}^{T} \sigma_{i} \Lambda_{i} K_{x i}+\sum_{i=1}^{m} \frac{\omega_{i}}{2} \sigma_{i} \tau_{i} k_{1 i}^{2}+\frac{\varrho_{1}}{2} \varsigma_{r i}^{T} \sigma_{i} \Theta_{i} \xi_{i}^{2} K_{r i}+\frac{\varrho_{2}}{2} \varsigma_{2} \chi \xi_{2}^{2}+2 \chi l_{3} . \\
&
\end{aligned}
$$

By applying Lemma 4, let

$$
\begin{aligned}
& y=\frac{2 \vartheta_{i}-1}{2 \vartheta_{i}} \sigma_{i} \widetilde{K}_{x i}^{T} \Lambda_{i} \widetilde{K}_{x i}, \\
& z=1, \\
& a=\alpha, \\
& b=1-\alpha, \\
& \mu=\frac{1}{\alpha},
\end{aligned}
$$

it has

$$
\left(\frac{2 \vartheta_{i}-1}{2 \vartheta_{i}} \sigma_{i} \widetilde{K}_{x i}^{T} \Lambda_{i} \widetilde{K}_{x i}\right)^{\alpha} \leq \frac{2 \vartheta_{i}-1}{2 \vartheta_{i}} \sigma_{i} \widetilde{K}_{x i}^{T} \Lambda_{i} \widetilde{K}_{x i}+\bar{\alpha},
$$

where $\bar{\alpha}=(1-\alpha) \alpha^{\alpha / 1-\alpha}$.

Similarly, we can conclude that

$$
\begin{gathered}
\left(\frac{2 \gamma_{i}-1}{2 \gamma_{i}} \sigma_{i} \widetilde{K}_{r i}^{T} \Theta_{i} \widetilde{K}_{r i}\right)^{\alpha} \leq \frac{2 \gamma_{i}-1}{2 \gamma_{i}} \sigma_{i} \widetilde{K}_{r i}^{T} \Theta_{i} \widetilde{K}_{r i}+\bar{\alpha}, \\
\left(\frac{2 \omega_{i}-1}{2 \omega_{i}} \sigma_{i} \tau_{i} \widetilde{k}_{1 i}^{2}\right)^{\alpha} \leq \frac{2 \omega_{i}-1}{2 \omega_{i}} \sigma_{i} \tau_{i} \widetilde{k}_{1 i}^{2}+\bar{\alpha} \\
\left(\frac{2 \varrho_{1}-1}{2 \varrho_{1}} \varsigma_{1} \chi \widetilde{\xi}_{1}^{2}\right)^{\alpha} \leq \frac{2 \varrho_{1}-1}{2 \varrho_{1}} \varsigma_{1} \chi \widetilde{\xi}_{1}^{2}+\bar{\alpha} \\
\left(\frac{2 \varrho_{2}-1}{2 \varrho_{2}} \varsigma_{2} \chi \widetilde{\xi}_{2}^{2}\right)^{\alpha} \leq \frac{2 \varrho_{2}-1}{2 \varrho_{2}} \varsigma_{2} \chi \widetilde{\xi}_{2}^{2}+\bar{\alpha} .
\end{gathered}
$$

Substituting (B.22)-(B.26) into (B.20) and applying Lemma 2, we have

$$
\begin{aligned}
\dot{V} \leq & -\sum_{i=1}^{m}\left(\frac{\left(2 \vartheta_{i}-1\right) \lambda_{\min }\left(\Lambda_{i}\right)}{\vartheta_{i} \lambda_{\max }\left(\Gamma_{i}^{-1}\right)}\right)^{\alpha}\left(\frac{1}{2} \sigma_{i} \widetilde{K}_{x i}^{T} \Gamma_{i}^{-1} \widetilde{K}_{x i}\right)^{\alpha} \\
& -\sum_{i=1}^{m}\left(\frac{\left(2 \gamma_{i}-1\right) \lambda_{\min }\left(\Theta_{i}\right)}{\gamma_{i} \lambda_{\max }\left(\Upsilon_{i}^{-1}\right)}\right)^{\alpha}\left(\frac{1}{2} \sigma_{i} \widetilde{K}_{r i}^{T} \Upsilon_{i}^{-1} \widetilde{K}_{r i}\right)^{\alpha} \\
& -\sum_{i=1}^{m}\left(\frac{\left(2 \omega_{i}-1\right) \mu_{i} \tau_{i}}{\omega_{i}}\right)^{\alpha}\left(\frac{1}{2} \sigma_{i} \mu_{i}^{-1} \widetilde{k}_{1 i}^{2}\right)^{\alpha} \\
& -\left(\frac{\left(2 \varrho_{1}-1\right) c_{1} \varsigma_{1}}{\varrho_{1}}\right)^{\alpha}\left(\frac{1}{2} c_{1}^{-1} \chi \tilde{\xi}_{1}^{2}\right)^{\alpha} \\
& -\left(\frac{\left(2 \varrho_{2}-1\right) c_{2} \varsigma_{2}}{\varrho_{2}}\right)^{\alpha}\left(\frac{1}{2} c_{2}^{-1} \chi \widetilde{\xi}_{2}^{2}\right)^{\alpha} \\
& -l_{2}\left(e^{T} P e\right)^{\alpha}+\sum_{i=1}^{m} \frac{\vartheta_{i}}{2} K_{x i}^{T} \sigma_{i} \Lambda_{i} K_{x i} \\
& \left.+\sum_{i=1}^{m}\left(\frac{1}{2} \sigma_{i} \mu_{i}^{-1} \widetilde{k}_{1 i}^{2}\right)^{\alpha}+\left(\frac{1}{2} c_{1}^{-1} \chi \widetilde{\xi}_{1}^{2}\right)^{\alpha}+\left(\frac{1}{2} c_{2}^{-1} \chi \widetilde{\xi}_{2}^{2}\right)^{\alpha}\right]+\bar{\psi} \\
& +\sum_{i=1}^{m} \frac{\gamma_{i}}{2} K_{r i}^{T} \sigma_{i} \Theta_{i} K_{r i}+\sum_{i=1}^{m} \frac{\omega_{i}}{2} \sigma_{i} \tau_{i} k_{1 i}^{2}+\frac{\varrho_{1}}{2} \varsigma_{1} \chi \xi_{1}^{2} \\
& +\frac{\varrho_{2}}{2} \varsigma_{2} \chi \xi_{2}^{2}+2 \chi l_{3}+(3 m+2) \bar{\alpha} \\
& -\left[\left(e^{T} P e\right)^{\alpha}+\sum_{i=1}^{m}\left(\frac{1}{2} \sigma_{i} \widetilde{K}_{x i}^{T} \Gamma_{i}^{-1} \widetilde{K}_{x i}\right)^{\alpha}+\sum_{i=1}^{m}\left(\frac{1}{2} \sigma_{i} \widetilde{K}_{r i}^{T} \Upsilon_{i}^{-1} \widetilde{K}_{r i}\right)^{\alpha}\right. \\
&
\end{aligned}
$$

where 


$$
\begin{aligned}
\eta= & \min \left\{l_{2},\left(\frac{\left(2 \vartheta_{i}-1\right) \lambda_{\min }\left(\Lambda_{i}\right)}{\vartheta_{i} \lambda_{\max }\left(\Gamma_{i}^{-1}\right)}\right)^{\alpha},\left(\frac{\left(2 \gamma_{i}-1\right) \lambda_{\min }\left(\Theta_{i}\right)}{\gamma_{i} \lambda_{\max }\left(\Upsilon_{i}^{-1}\right)}\right)^{\alpha},\right. \\
& \left.\left(\frac{\left(2 \omega_{i}-1\right) \mu_{i} \tau_{i}}{\omega_{i}}\right)^{\alpha},\left(\frac{\left(2 \varrho_{1}-1\right) c_{1} \varsigma_{1}}{\varrho_{1}}\right)^{\alpha},\left(\frac{\left(2 \varrho_{2}-1\right) c_{2} \varsigma_{2}}{\varrho_{2}}\right)^{\alpha}\right\}, \\
\bar{\psi}= & \sum_{i=1}^{m} \frac{\vartheta_{i}}{2} K_{x i}^{T} \sigma_{i} \Lambda_{i} K_{x i}+\sum_{i=1}^{m} \frac{\gamma_{i}}{2} K_{r i}^{T} \sigma_{i} \Theta_{i} K_{r i} \\
& +\sum_{i=1}^{m} \frac{\omega_{i}}{2} \sigma_{i} \tau_{i} k_{1 i}^{2}+\frac{\varrho_{1}}{2} \varsigma_{1} \chi \xi_{1}^{2}+\frac{\varrho_{2}}{2} \varsigma_{2} \chi \xi_{2}^{2}+2 \chi l_{3}+(3 m+2) \bar{\alpha} .
\end{aligned}
$$

Furthermore, according to Lemma 1, one has

$$
\dot{V} \leq-\eta V^{\alpha}+\bar{\psi}
$$

Therefore, according to Lemma 5, the decrease of $\mathrm{V}$ can drive the trajectories of the closed-loop system into $V^{\alpha} \leq(\bar{\psi} /(1-\imath) \eta)$ in a finite time $T_{r}$ with

$$
T_{r} \leq \frac{V^{1-\alpha}(0)}{\eta \iota(1-\alpha)},
$$

where $0<\iota<1$.

This completes the proof.

\section{Data Availability}

The data used to support the findings of this study are available from the corresponding author upon request.

\section{Conflicts of Interest}

The authors declare that there are no conflicts of interest regarding the publication of this paper.

\section{Acknowledgments}

This work was supported by the National Natural Science Foundation of China under grant nos. 61573161 and 61873319.

\section{References}

[1] Z. Mao and B. Jiang, "Fault identification and fault-tolerant control for a class of networked control systems," International Journal of Innovative Computing, Information and Control, vol. 3, no. 5, pp. 1121-1130, 2007.

[2] C. Peng, E. G. Tian, and T. C. Yang, "Robust fault-tolerant control of networked control systems with stochastic actuator failure," IET Control Theory \& Applications, vol. 4, no. 12, pp. 3003-3011, 2010.

[3] Z. Chen, Q. Chen, X. He, and M. Sun, "Adaptive finite-time command filtered fault-tolerant control for uncertain spacecraft with prescribed performance," Complexity, vol. 2018, Article ID 4912483, 12 pages, 2018.

[4] S. Park, J. Bae, Y. Kim, and S. Kim, "Fault tolerant flight control system for the tilt-rotor UAV," Journal of the Franklin Institute, vol. 350, no. 9, pp. 2535-2559, 2013.
[5] J. Niu, F. Chen, and G. Tao, "Nonlinear fuzzy fault-tolerant control of hypersonic flight vehicle with parametric uncertainty and actuator fault," Nonlinear Dynamics, vol. 92, no. 7, pp. 1299-1315, 2018.

[6] G. Yang, J. L. Wang, and Y. C. Soh, "Reliable $H_{\infty}$ controller design for linear systems," Automatica, vol. 37, no. 5, pp. 717-725, 2001.

[7] S. M. Tabatabaeipour, R. Izadi-Zamanabadi, T. Bak, and A. P. Ravn, "Passive fault-tolerant control of discrete time piecewise affine systems against actuator faults," International Journal of Systems Science, vol. 43, no. 11, pp. 1985-1997, 2012.

[8] R. Wang and J. Wang, "Passive actuator fault-tolerant control for a class of overactuated nonlinear systems and applications to electric vehicles," IEEE Transactions on Vehicular Technology, vol. 62, no. 3, pp. 972-985, 2013.

[9] A. Nasiri, S. K. Nguang, A. Swain, and D. Almakhles, "Passive actuator fault tolerant control for a class of MIMO nonlinear systems with uncertainties," International Journal of Control, vol. 92, no. 3, pp. 693-704, 2019.

[10] C. P. Tan and C. Edwards, "Sliding mode observers for robust detection and reconstruction of actuator and sensor faults," International Journal of Robust and Nonlinear Control, vol. 13, no. 5, pp. 443-463, 2003.

[11] L. I. Allerhand and U. Shaked, "Robust switching-based fault tolerant control," IEEE Transactions on Automatic Control, vol. 60, no. 8, pp. 2272-2276, 2015.

[12] Z. Gao, P. Cheng, M. Qian, G. Jiang, and J. Lin, “Active faulttolerant control approach design for rigid spacecraft with multiple actuator faults," Proceedings of the Institution of Mechanical Engineers, Part I: Journal of Systems and Control Engineering, vol. 232, no. 10, pp. 1365-1378, 2018.

[13] C.-C. Chen, S. S.-D. Xu, and Y.-W. Liang, "Study of nonlinear integral sliding mode fault-tolerant control," IEEE/ASME Transactions on Mechatronics, vol. 21, no. 2, pp. 1160-1168, 2016.

[14] Z. Gao and P. J. Antsaklis, "Stability of the pseudo-inverse method for reconfigurable control systems," International Journal of Control, vol. 53, no. 3, pp. 717-729, 1991.

[15] N. Esfahani and K. Khorasani, "A distributed model predictive control (MPC) fault reconfiguration strategy for formation flying satellites," International Journal of Control, vol. 89, no. 5, pp. 960-983, 2016.

[16] L.-B. Wu, G.-H. Yang, and D. Ye, "Robust adaptive faulttolerant control for linear systems with actuator failures and mismatched parameter uncertainties," IET Control Theory \& Applications, vol. 8, no. 6, pp. 441-449, 2014.

[17] Y. Ma, B. Jiang, G. Tao, and H. Yang, "Adaptive direct compensation control scheme for spacecraft with multiple actuator faults," Journal of Guidance, Control, and Dynamics, vol. 42, no. 4, pp. 923-930, 2019.

[18] S. Xiao and J. Dong, "Robust adaptive fault-tolerant tracking control for uncertain linear systems with time-varying performance bounds," International Journal of Robust and Nonlinear Control, vol. 29, no. 4, pp. 849-866, 2019.

[19] B. Liu, M. Saif, and H. Fan, "Adaptive fault tolerant control of a half-car active suspension systems subject to random actuator failures," IEEE/ASME Transactions on Mechatronics, vol. 21, no. 6, pp. 2847-2857, 2016.

[20] W. Wang, C. Wen, and G. Yang, "Stability analysis of decentralized adaptive backstepping control systems with actuator failures," Journal of Systems Science and Complexity, vol. 22, no. 1, pp. 109-121, 2009.

[21] X. Tang, G. Tao, L. Wang, and J. A. Stankovic, "Robust and adaptive actuator failure compensation designs for a rocket 
fairing structural-acoustic model," IEEE Transactions on Aerospace and Electronic Systems, vol. 40, no. 4, pp. 13591366, 2004.

[22] X. Jin, J. Qin, Y. Shi, and W. X. Zheng, “Auxiliary fault tolerant control with actuator amplitude saturation and limited rate," IEEE Transactions on Systems, Man, and Cybernetics: Systems, vol. 48, no. 10, pp. 1816-1825, 2017.

[23] X. Jin, S. Lü, J. Qin, and W. X. Zheng, "Auxiliary constrained control of a class of fault-tolerant systems," IEEE Transactions on Systems, Man, and Cybernetics: Systems, pp. 1-12, 2019.

[24] C. Wang, C. Wen, and Y. Lin, "Adaptive actuator failure compensation for a class of nonlinear systems with unknown control direction," IEEE Transactions on Automatic Control, vol. 62, no. 1, pp. 385-392, 2017.

[25] B. Liu, L. Liu, Y. Wang, and H. Fan, "Stabilization of nonlinear uncertain systems with stochastic actuator failures and timevarying delay," International Journal of Robust and Nonlinear Control, vol. 26, no. 9, pp. 1825-1840, 2016.

[26] C.-H. Xie and G.-H. Yang, "Model-free fault-tolerant control approach for uncertain state-constrained linear systems with actuator faults," International Journal of Adaptive Control and Signal Processing, vol. 31, no. 2, pp. 223-239, 2017.

[27] A. Ben Brahim, S. Dhahri, F. Ben Hmida, and A. Sellami, "Multiplicative fault estimation-based adaptive sliding mode fault-tolerant control design for nonlinear systems," Complexity, vol. 2018, Article ID 1462594, 15 pages, 2018.

[28] S. Xiao and J. Dong, "Robust adaptive fault-tolerant tracking control for uncertain linear systems with actuator failures based on the closed-loop reference model," IEEE Transactions on Systems, Man, and Cybernetics: Systems, pp. 1-8, 2018.

[29] S. M. Joshi and P. Patre, "Direct model reference adaptive control with actuator failures and sensor bias," Journal of Guidance, Control, and Dynamics, vol. 37, no. 1, pp. 312-317, 2014.

[30] J. Wang, J. Du, F. Chen, B. Jiang, and G. Tao, "Direct adaptive control scheme for a quadrotor helicopter with actuator failures via quantum logic," Journal of Aerospace Engineering, vol. 29, no. 5, pp. 1-7, 2016.

[31] L.-B. Wu and G.-H. Yang, "Robust adaptive fault-tolerant tracking control of multiple time-delays systems with mismatched parameter uncertainties and actuator failures," International Journal of Robust and Nonlinear Control, vol. 25, no. 16, pp. 2922-2938, 2015.

[32] G. Song and G. Tao, "Adaptive state-feedback control with sensor failure compensation for asymptotic output tracking," International Journal of Adaptive Control and Signal Processing, vol. 33, no. 1, pp. 130-156, 2019.

[33] J. Yu, P. Shi, and L. Zhao, "Finite-time command filtered backstepping control for a class of nonlinear systems," Automatica, vol. 92, pp. 173-180, 2018.

[34] L. Zhao, J. Yu, and P. Shi, "Command filtered backsteppingbased attitude containment control for spacecraft formation," IEEE Transactions on Systems, Man, and Cybernetics: Systems, pp. 1-10, 2019.

[35] J. Wang, C. Zhang, S. Li, J. Yang, and Q. Li, "Finite-time output feedback control for PWM-based DC-DC buck power converters of current sensorless mode," IEEE Transactions on Control Systems Technology, vol. 25, no. 4, pp. 1359-1371, 2016.

[36] Z. Zhu, Y. Xia, and M. Fu, "Attitude stabilization of rigid spacecraft with finite-time convergence," International Journal of Robust and Nonlinear Control, vol. 21, no. 6, pp. 686-702, 2011.
[37] Y. Zhang, Y. Liu, and L. Liu, “Adaptive finite-time NN control for 3-DOF active suspension systems with displacement constraints," IEEE Access, vol. 7, pp. 13577-13588, 2019.

[38] F. Wang, X. Zhang, B. Chen, C. Lin, X. Li, and J. Zhang, "Adaptive finite-time tracking control of switched nonlinear systems," Information Sciences, vol. 421, pp. 126-135, 2017.

[39] X. Huo, Q. Hu, and B. Xiao, "Finite-time fault tolerant attitude stabilization control for rigid spacecraft," ISA Transactions, vol. 53, no. 2, pp. 241-250, 2014.

[40] X.-y. Zhang, Q. Zong, B.-1. Tian, S.-k. Shao, and W.-j. Liu, "Finite-time fault estimation and fault-tolerant control for rigid spacecraft," Journal of Aerospace Engineering, vol. 31, no. 6, Article ID 04018091, 2018.

[41] Q. Shen, D. Wang, S. Zhu, and K. Poh, "Finite-time faulttolerant attitude stabilization for spacecraft with actuator saturation," IEEE Transactions on Aerospace and Electronic Systems, vol. 51, no. 3, pp. 2390-2405, 2015.

[42] X.-Z. Jin, S.-F. Wang, G.-H. Yang, and D. Ye, "Robust adaptive hierarchical insensitive tracking control of a class of leader-follower agents," Information Sciences, vol. 406-407, pp. 234-247, 2017.

[43] S. P. Bhat and D. S. Bernstein, "Finite-time stability of continuous autonomous systems," SIAM Journal on Control and Optimization, vol. 38, no. 3, pp. 751-766, 2000.

[44] H. G. Harold, J. E. Littlewood, and G. Polya, Inequalities, Cambridge University Press, Cambridge, UK, 1952.

[45] R. A. Horn and C. R. Johnson, Matrix Analysis, Cambridge University Press, Cambridge, UK, 1990.

[46] C. Qian and W. Lin, "Non-lipschitz continuous stabilizers for nonlinear systems with uncontrollable unstable linearization," Systems \& Control Letters, vol. 42, no. 3, pp. 185-200, 2001.

[47] W. Siwakosit and R. A. Hess, "Multi-input/multi-output reconfigurable flight control design," Journal of Guidance, Control, and Dynamics, vol. 24, no. 6, pp. 1079-1088, 2001.

[48] R. Lamouchi, T. Raïssi, M. Amairi, and M. Aoun, "Interval observer framework for fault-tolerant control of linear parameter-varying systems," International Journal of Control, vol. 91, no. 3, pp. 524-533, 2018.

[49] X.-Z. Jin, Y.-G. He, and Y.-G. He, "Finite-time robust faulttolerant control against actuator faults and saturations," IET Control Theory \& Applications, vol. 11, no. 4, pp. 550-556, 2017.

[50] S. M. Joshi, P. Patre, and G. Tao, "Adaptive control of systems with actuator failures using an adaptive reference model," Journal of Guidance, Control, and Dynamics, vol. 35, no. 3, pp. 938-949, 2012.

[51] S. Chen, G. Tao, and S. M. Joshi, "On matching conditions for adaptive state tracking control of systems with actuator failures," IEEE Transactions on Automatic Control, vol. 47, no. 3, pp. 473-478, 2002. 


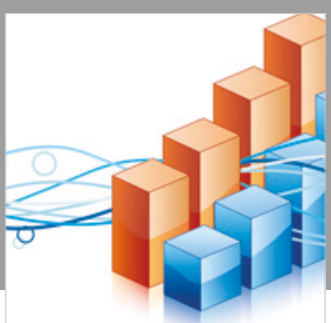

Advances in

Operations Research

\section{-n-m}
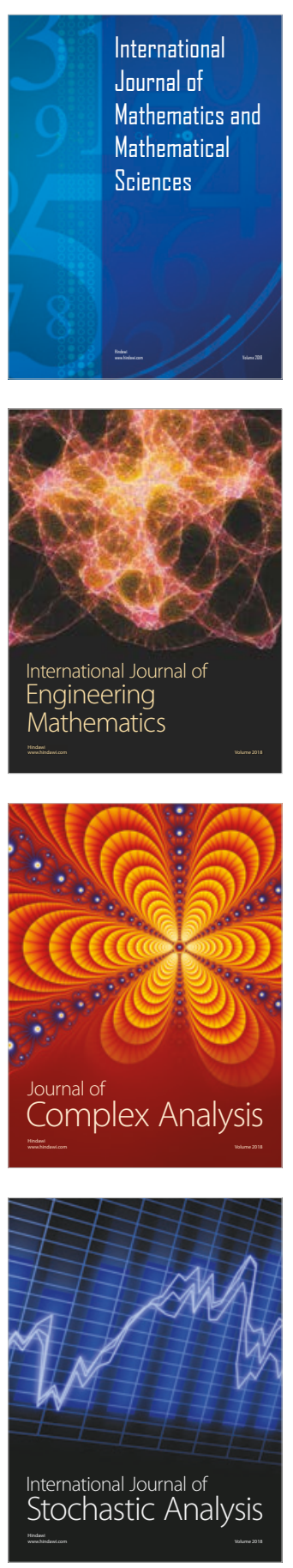
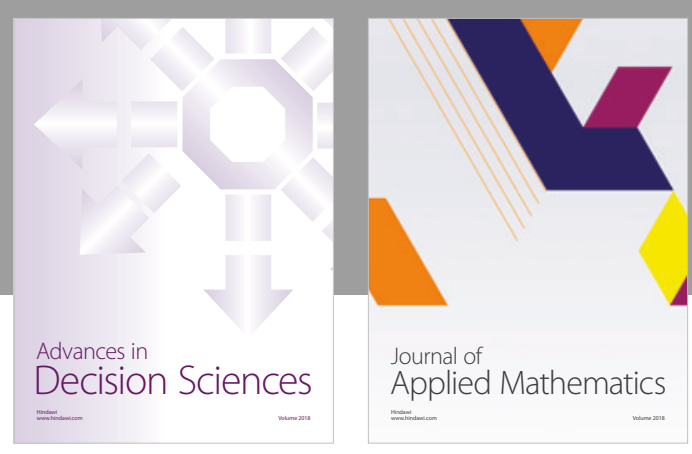

Journal of

Applied Mathematics
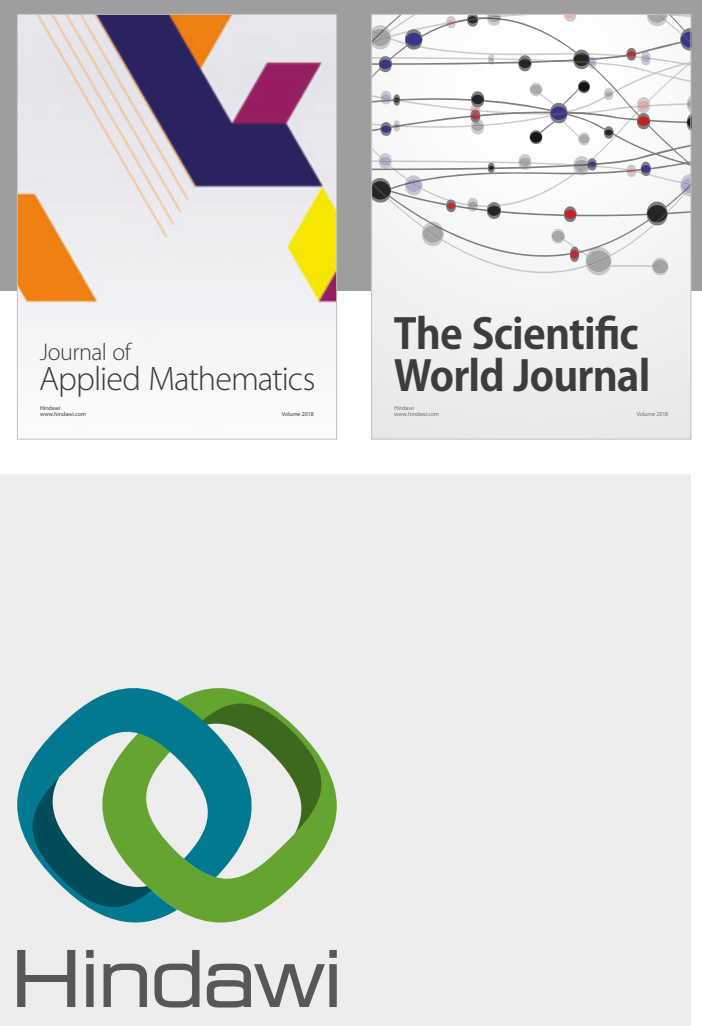

Submit your manuscripts at

www.hindawi.com

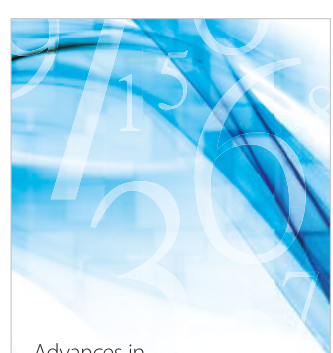

Advances in
Numerical Analysis
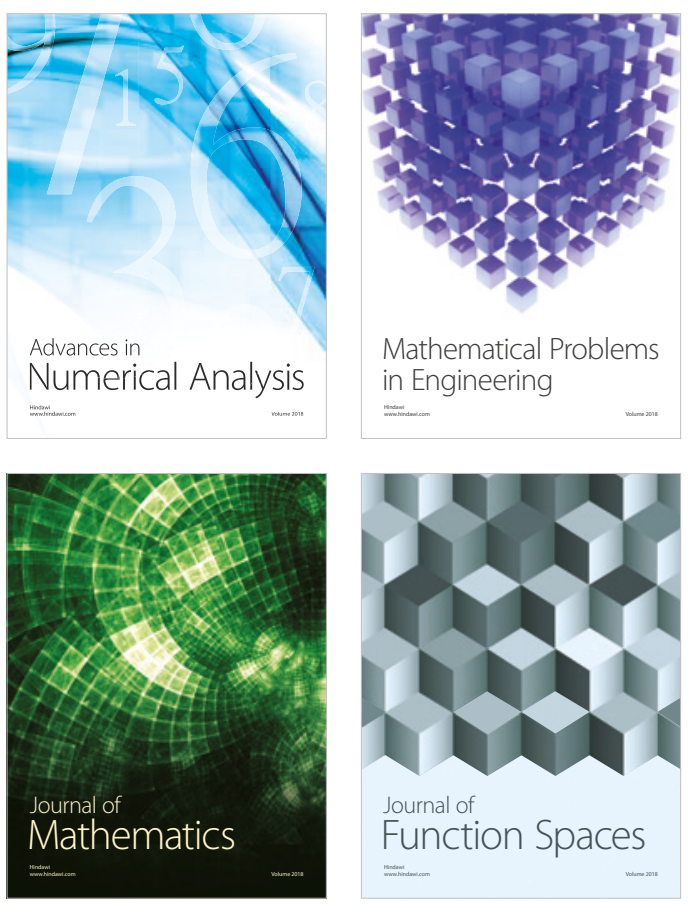

Mathematical Problems in Engineering

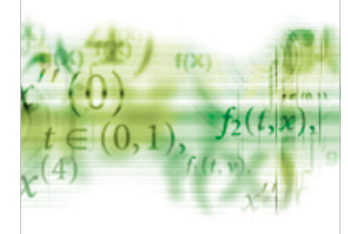

International Journal of

Differential Equations

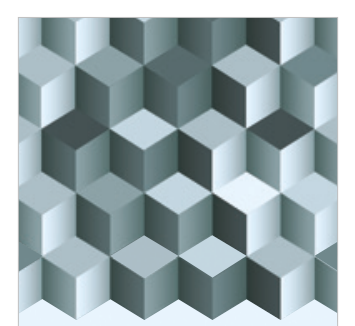

Journal of

Function Spaces
The Scientific

World Journal

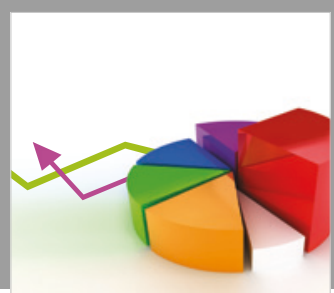

Journal of

Probability and Statistics
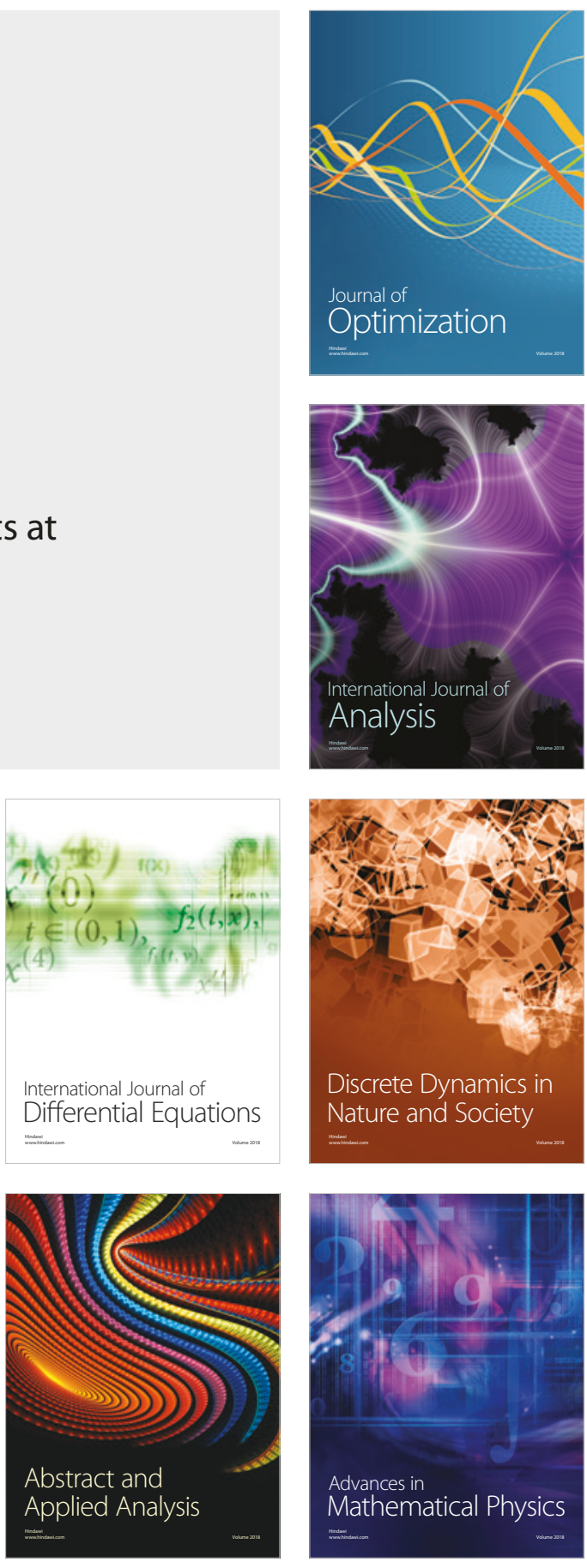